\title{
Metodología para cálculo de seeing en el Observatorio Astronómico Centroamericano de Suyapa, Tegucigalpa, Honduras
}

\author{
Roberto Schöngarth \\ Observatorio Astronómico Centroamericano de Suyapa \\ Universidad Nacional Autónoma de Honduras \\ robertoschongarth@yahoo.com
}

\begin{abstract}
RESUMEN
El presente trabajo propone una metodología a ser utilizada para la caracterización de las condiciones de observación en el Observatorio Astronómico Centroamericano de Suyapa OACS, particularmente en lo relacionado a la medición del seeing. Se utiliza el análisis de imágenes píxel por píxel tratando de estimar el seeing a través del FWHM, utilizando como límite superior el píxel de máxima intensidad de cada una de las imágenes de la estrella Zeta Geminorum que sirvió para hacer el estudio, y cuyo límite inferior es el promedio de intensidad del cielo de fondo. Para lograr mayor precisión en la estimación se promediaron los valores estimados de un grupo de 10 imágenes y a partir de ahí inferir un intervalo de confianza del 95\% para la estimación del seeing el cual para el presente trabajó resulto ser de 5.2332-5.8448 segundos de arco el cual es un valor de seeing relativamente alto (condiciones regulares de observación) respecto a los 2 ó a lo sumo 3 segundos de arco recomendados para observaciones con un buen cielo.
\end{abstract}

Palabras clave: Seeing, Escala de Pickering, FWHM, refracción, disco de Airy, resolución telescopio.

\begin{abstract}
The present work proposes a methodology to being used for the characterization of the conditions of observation in the Central American Suyapa Astronomical Observatory CASAO, particularly related to the measurement of the seeing. The analysis of images uses pixel by pixel, trying to estimate the seeing through the FWHM, using as top limit the pixel of maximum intensity of each one of the images of the major Zeta Geminorum that served to do the study, and whose low limit is the average of intensity of the background sky. To achieve major precision in the estimation there were divided equally the values estimated of a group of 10 images and from there inferring a confidence interval of $95 \%$ for the estimation of the seeing. For the present, this value worked turn out to be of 5.2332-5.8448 second of arch which is a value of seeing relatively high (regular conditions of observation) with regard to the 2 or at most 3 seconds of arch recommended for observations with a good sky.
\end{abstract}

Key words: Seeing, Pickering scale, FWHM, refraction, Airy disk, telescope resolution. 


\section{INTRODUCCIÓN}

La caracterización del cielo de un lugar es esencial para poder determinar que tanto se puede observar y cuál podría ser la calidad de las observaciones según el tipo de tecnología de que se disponga. Las perturbaciones en la atmósfera hacen variar la calidad de imágenes en tierra pues éstas se ven afectadas por cambios de temperatura en capas superiores y por la topografía del lugar, perturbaciones que pueden ser medidas en lo que se conoce como seeing. Por tanto hay que medir con el equipo disponible la magnitud de estas oscilaciones.

Cuál es el mejor método y en qué escala se puede medir el seeing es el análisis que se hará en el presente trabajo para posteriormente ser aplicado para determinar las condiciones de observación en el Observatorio Astronómico Centroamericano de Suyapa (OACS). El objetivo de este trabajo ha sido proponer un método de análisis del seeing para medir las condiciones de la atmósfera para observación astronómica desde el OACS.

\section{Medicion del seeing utilizando escalas generales}

El seeing es una medida que se ha tratado de determinar desde hace más de un siglo para establecer las condiciones de observación. Es por ello que fue ampliamente utilizada la Escala de Pickering, ya no muy vigente en el presente dados los avances tecnológicos en la observación.

La Escala de Pickering propone medir, en una escala del 1 al 10, la calidad del cielo observando la turbulencia de una estrella de referencia observada, tal y como se presenta en la figura 1. En esta figura se muestra de manera clara cómo varía la calidad de una imagen dependiendo de las condiciones atmosféricas pues, si se analiza con mayor detalle, la escala de Pickering observa la calidad del disco central y el diámetro de la imagen difractada. Cuando la calidad es de 10 la imagen se presenta completamente estática, con el disco de Airy muy bien definido; al ir reduciendo la calidad, los patrones alrededor de punto central comienzan a crecer y a mostrar arcos. Cuando la calidad ya es tan baja como 4 , el disco central es difuso. Cuando la calidad es de 1 la imagen de la estrella es generalmente del doble del diámetro del tercer anillo de difracción.

FIGURA 1. Escala de medición de seeing de Pickering

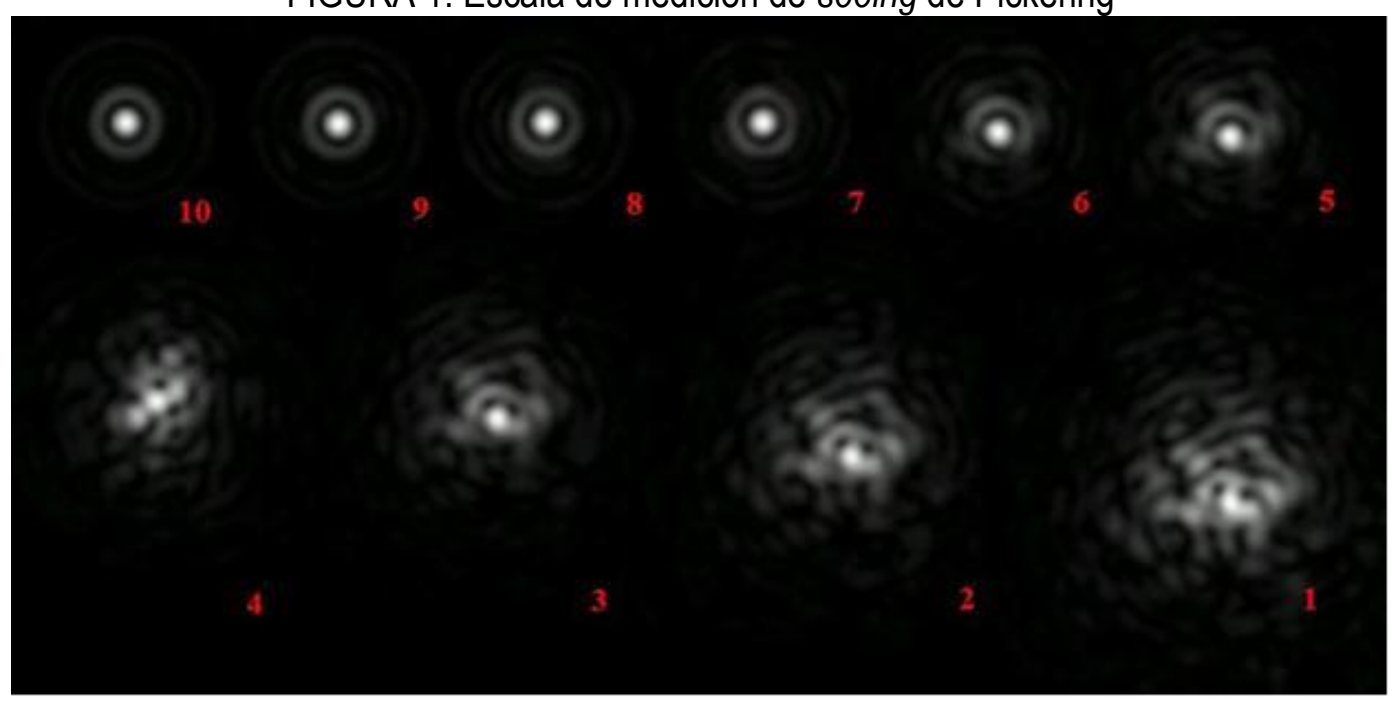


Escalas alternativas como la de Antoniadi también son muy superficiales y no cubren las expectativas de una medición precisa de calidad de cielo. Corresponde a una escala de I a V, donde I indica visibilidad perfecta y $\mathrm{V}$ muy pobre visibilidad.

Un estudio de la calidad de cielo cerca de Sydney, Australia, efectuado por J.W. O'Byrne en 1984, arrojó resultados interesantes: las noches con mejor seeing en promedio corresponden a las noches de verano; la relación entre seeing y velocidad del viento no es simple, pero generalmente un buen seeing está asociado a lentas velocidades de viento; hay cierta evidencia que hay buenas condiciones de seeing cuando hay nubes delgadas o niebla presentes.

\section{Medicion del seeing utilizando FWHM}

La medición del seeing toma en cuenta cada uno de los filtros disponibles, por lo que para hacer el análisis en el OACS deberá trabajarse con cada uno de los 5 filtros de que se dispone: U, B, V R, I.

Si las perturbaciones en la atmósfera fuesen nulas, el tamaño de la estrella a través del telescopio sería igual al que determina su disco de difracción. Las perturbaciones de la atmósfera hacen crecer en apariencia a este disco, y esto es lo que trata de medir el FWHM (full width at half máximum).

El FWHM es el ancho de la función, o sea su proyección al eje horizontal, que está expresado en segundos de arco, donde el flujo toma la mitad del valor máximo. Lo importante es considerar que este valor es igual para todas las estrellas tomadas en una misma imagen. Obsérvese la figura 2, donde el FWHM muestra el ancho de la función en la mitad que corresponde al pico de intensidad de cada una de las dos estrellas y el valor del cielo de fondo. En este caso particular este valor es de aparentemente 2.5 segundos de arco. Por lo tanto el seeing es el tamaño angular de la estrella medida al punto FWHM.

FIGURA 2. Medición del FWHM para dos estrellas diferentes en el mismo instante. El FWHM es igual.

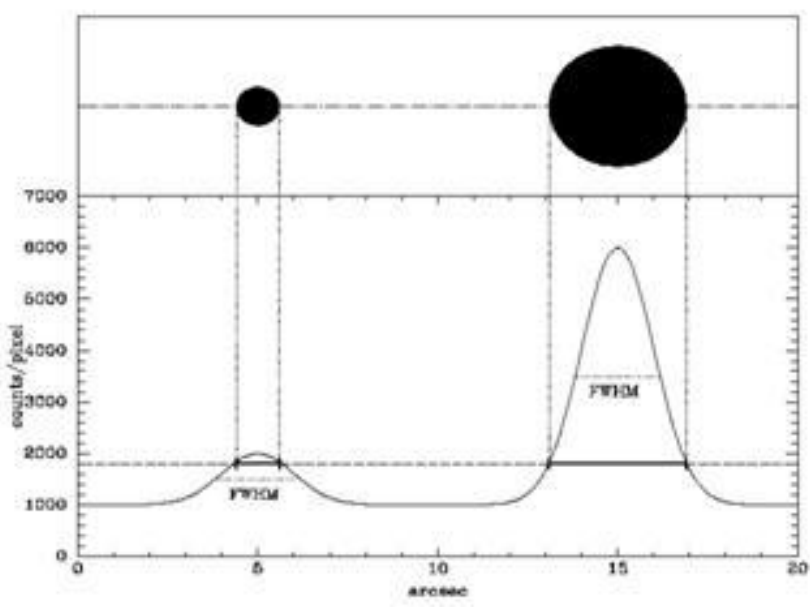

\section{METODOLOGIA}

Previo a obtener datos prácticos para hacer un estudio pormenorizado del seeing, día a día, es conveniente mostrar un procedimiento que pudiera utilizarse para el cálculo del FWHM con un dato histórico. La metodología a utilizar puede resumirse en los siguientes pasos:

- El primer paso consiste en seleccionar las imágenes a analizar para estimar el seeing durante la noche de observación. Es recomendable utilizar un número de imágenes significativo como muestra para poder obtener una estimación lo más fiable posible. 
- Analizar las imágenes utilizando la metodología del FWHM. Hay que localizar el pixel de mayor intensidad y además estimar la intensidad del cielo de fondo. Para ello se hará uso de un programa especializado que muestre la intensidad de cada uno de los píxeles. Para el cielo de fondo se recomienda usar una muestra aleatoria de varios píxeles y promediar su valor. El valor medio que sirve como referencia en el FWHM se calcula con (intensidad máxima - intensidad de fondo) / 2 + intensidad de fondo, lo que al simplificar la fórmula resulta en:

(intensidad máxima + intensidad de fondo) / 2

- Estimar el número de píxeles de la imagen que supera el valor medio estimado. Como se asume una simetría circular en la imagen se supondrá que en cualquier dirección la cantidad de píxeles que supere el valor medio debe ser la misma.

- Calcular el valor de seeing en segundos de arco, encontrando la ecuación que relaciona píxeles y segundos de arco, la cual es dependiente de la longitud focal del objetivo y el tamaño del píxel.

- Se calcula el valor del seeing para todas las imágenes tomadas hacia el mismo objeto. Al calcular la media y la desviación estándar de la muestra es posible inferir un intervalo de confianza para tener una aproximación significativa para el seeing.

\section{RESULTADOS}

- En este estudio se utilizaron diez imágenes tomadas desde el Observatorio Astronómico Centroamericano de Suyapa el 6 de febrero de 2007 por Isaac Zablah. La figura 3 expone de forma ampliada la primera fotografía tomada mostrando la estrella Zeta Geminorum. La imagen se tomó desde el telescopio Meade de 16". Para poder observarla con mayor detalle aquí se muestra la imagen con un zoom de 16x utilizando el visor de archivos fit ds9 utilizado en Linux.

Figura 3: Estrella Zeta Geminorum. Imagen tomada el 6 de febrero 2007 por Isaac Zablah desde OACS. Telecopio Meade 16", filtro V.

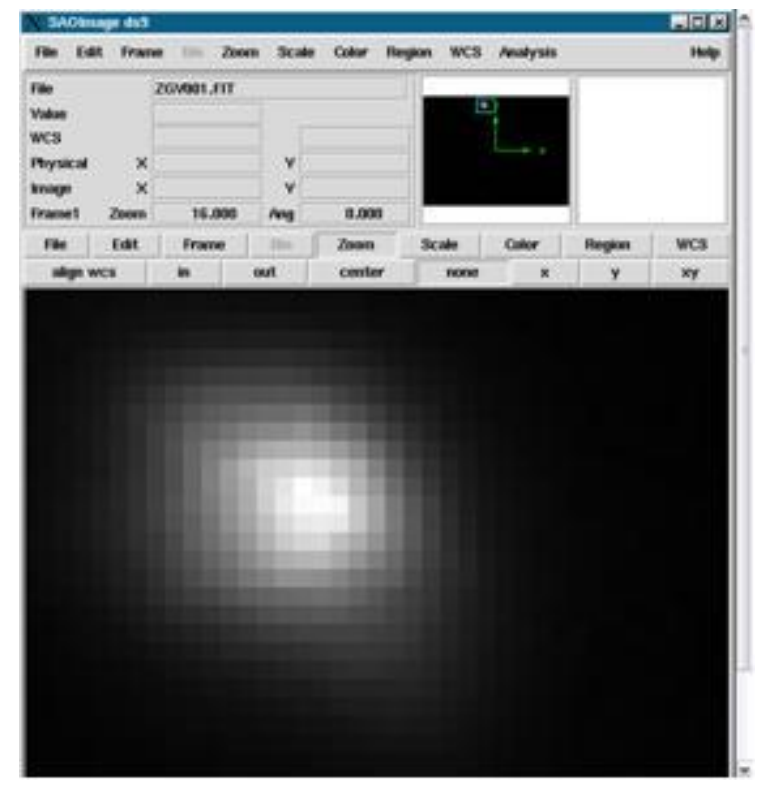


- Para el análisis aquí se dio seguimiento a la primera de las diez imágenes tomadas durante esa noche, pero cabe agregar que el procedimiento se repitió para las nueve imágenes restantes. Para utilizar la metodología de FWHM encontramos el píxel de mayor intensidad, que en el presente caso consta de 10036 cuentas, y la intensidad el cielo de fondo, tomando una muestra de píxeles oscuros alejados de la imagen de la estrella, que indica un promedio de 191 cuentas la presente imagen. El valor promedio total, $(10036+191) / 2=5113$, es el valor de referencia que se utilizó para el cálculo del seeing.

- Como siguiente paso se estimó el número de píxeles de la imagen que supera el valor medio estimado. Para aclarar esto se comenzó analizando una línea horizontal que pasa a través del píxel máximo, donde las intensidades por píxel observadas serán las que se muestran en la figura 4. A manera de ejemplo obsérvese el número de píxeles que supera una intensidad de 5113 , valor de referencia. Para este caso particular el número de píxeles es de 9 , cuyo equivalente en segundos de arco es el seeing (como se explica más adelante, el equivalente a los 9 píxeles es de 4.5675 segundos de arco). Antes de esta operación de equivalencia píxeles-segundos de arco, recordamos que es ideal analizar el seeing en todas direcciones de la estrella y no sólo de la línea horizontal antes trazada. Por lo tanto el siguiente paso fue contabilizar todos los píxeles de la figura que superan las 5113 cuentas. Por simetría circular esperábamos que este valor fuera igual sin importar la dirección en que se mida.

FIGURA 4: Intensidad por píxel de imagen, con máxima intensidad centrada

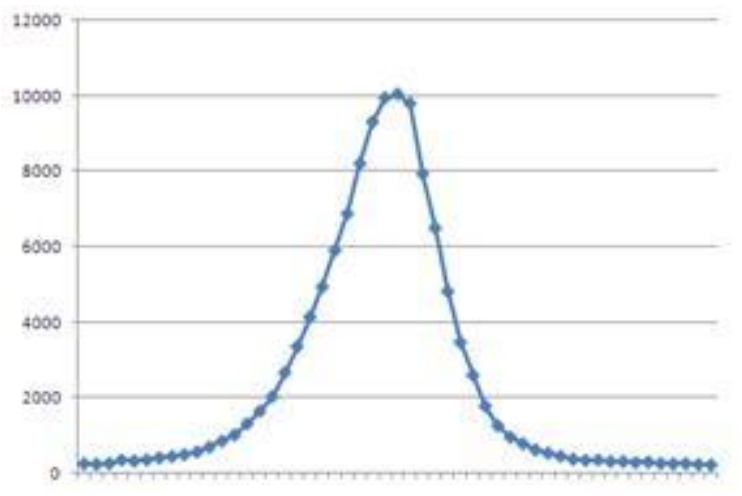

Se aprovechó este hecho para hacer el cálculo precisamente en todas direcciones y así estimar un valor promediado. Se contó por lo tanto el número de píxeles que superan el valor del FWHM y se calculó el diámetro promedio. En este ejemplo fue de 71 píxeles los que superan las 5113 cuentas, o sea un círculo de 71 píxeles.

- A continuación se hizo una estimación promedio del diámetro del círculo utilizando el valor de píxeles encontrados que superan el valor FWHM que representan el área del círculo. El diámetro del círculo encontrado más que todo da un promedio del ancho del FWHM en todas direcciones, lo que se logra despejando de la siguiente fórmula:

$$
\begin{gathered}
\text { Área del círculo }=\pi r^{2}=\pi(d / 2)^{2} \\
d=2 \sqrt{ }(A / \pi) \\
d=2 \sqrt{ }(71 / \pi)=9.5079 \text { píxeles }
\end{gathered}
$$


Por lo tanto, si se promedia en cualquier dirección respecto al píxel de mayor intensidad, el ancho de píxeles para medir el seeing es de 9.5079.

- Por supuesto, el seeing debe estar medido en segundos de arco, por lo que hace falta hacer el análisis de placa para poder determinar una relación entre píxel y tamaño en segundos de arco.

Esta relación se logró utilizando los siguientes datos:

Longitud focal objetivo de telescopio $=4064 \mathrm{~mm}$

Tamaño de píxel $=10 \mu \mathrm{m}=0.01 \mathrm{~mm}$

La escala de placa para el telescopio es de $\Delta \alpha / \Delta s=206,265 " / 4,064 \mathrm{~mm}=50.75 " / \mathrm{mm}$.

Por lo tanto el equivalente angular de un píxel es de $50.75 " / \mathrm{mm} *(0.01 \mathrm{~mm})=0.5075 "$.

El seeing entonces fue el resultado de multiplicar los píxeles promedio por el equivalente angular del píxel:

\section{SEEING $=9.5079$ píxeles * $0.5075 " / p i ́ x e l=4.8253 "$}

- Se repitió el procedimiento para las nueve imágenes restantes. Los resultados obtenidos para cada una de las diez imágenes, pareando la mayor intensidad de píxel para cada imagen respecto al seeing estimado después de haber hecho los cálculos correspondientes utilizando la fórmula mostrada en el paso anterior, resultaron en el diagrama de dispersión mostrado en la figura 5.

FIGURA 5: Diagrama de dispersión con la relación entre el píxel de mayor intensidad y el seeing en segundos de arco medido para cada imagen.

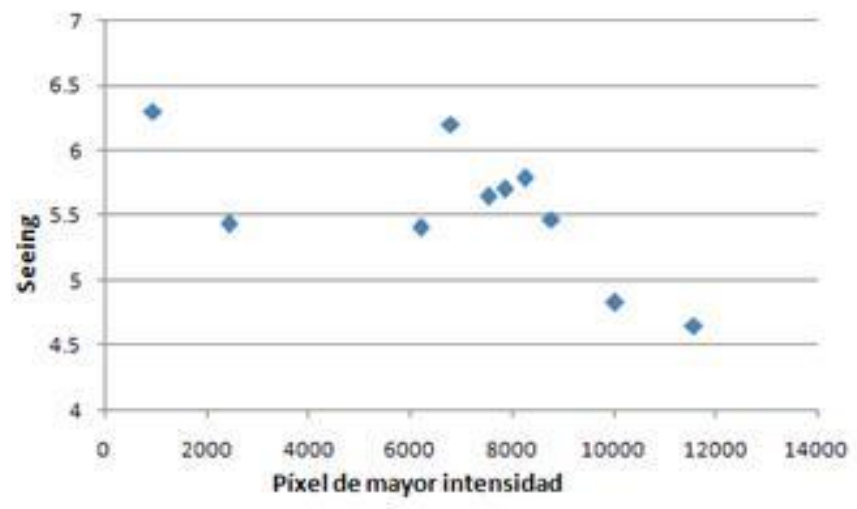

Como el tamaño de muestra es de diez, se calculó una estimación del intervalo de confianza para el seeing de la siguiente manera:

$$
\mathrm{IC}=\overline{\mathrm{X}} \pm \mathrm{Zs} / \mathrm{Vn}_{\mathrm{n}}
$$

$\underline{X}$ es el valor de la media muestral, $Z=1.96$ para un nivel de confianza del $95 \%$, s es el valor de la desviación estándar muestral y n es el tamaño de la muestra, por lo que al sustituir resultó:

$$
\mathrm{IC}=5.5390 \pm 1.96 * 0.4933 / \sqrt{ } 10
$$

\section{$5.2332 \leq$ seeing $\leq 5.8448$}

- Habiendo ya calculado el seeing, no es necesario regresar a la escala de Pickering a menos que el observador esté adquiriendo la experiencia en esta interpretación. Para ello la figura 6 muestra una equivalencia teórica entre seeing y las condiciones del cielo que, para el ejemplo que se acaba de proponer, indica que la escala de Pickering evalúa este cielo con un 4, o sea que las condiciones estaban entre medianas a buenas. 
FIGURA 6. Equivalencia teórica entre la escala de Pickering y el seeing medido a través del FWHM.

\begin{tabular}{|c|c|c|c|c|c|c|c|c|c|}
\hline \multicolumn{10}{|c|}{$\begin{array}{l}\text { Escala Pickering cotas en arc.seg. } \\
\text { Condiciones de malas a medianas de medianas a buenas de buenas a excelentes }\end{array}$} \\
\hline 1 & 2 & 3 & 4 & 5 & 6 & 7 & 8 & 9 & 10 \\
\hline \multicolumn{10}{|c|}{ Valores del SEEING en su punto FWHM, para cada nivel de la escala } \\
\hline 13,19 & 10,21 & 7,91 & 6,12 & 4,74 & 3,67 & 2,84 & 2,20 & 1,70 & 1,32 \\
\hline
\end{tabular}

Fuente: www.invlumer.e.telefonica.net/Planificando/SEEING/Escala_SEEING.htm

\section{CONCLUSIONES}

El procedimiento del cálculo del seeing mediante el FWHM es mucho más preciso dada la precisión de las cámaras CCD y la disponibilidad de software variado que permite analizar píxel por píxel la intensidad y características de una imagen.

Ello ha llevado a poder prescindir de metodologías anteriores las cuales no eran tan precisas y someramente daban una idea de la calidad del cielo sin llevar a un valor puntual como, por ejemplo, 5.5390 segundos de arco como el estudio que se realizó.

\section{RECOMENDACIONES}

- El estudio indica que debe tomarse un número de datos que como mínimo sean de una decena. Esto más allá de mejorar la imagen ya procesada al final con un programa como por ejemplo IRAF, permite también tener una aproximación precisa del valor de cada píxel y por lo tanto el valor final del seeing.

- Si bien el valor del FWHM debe ser la misma para cualquier estrella en la misma imagen sin importar su intensidad, para tener mejor precisión es recomendable utilizar imágenes cuya intensidad sea relativamente alta, obviamente cuidando el hecho de no saturar la imagen en ninguno de sus puntos.

- En cada observación debe hacerse una estimación del seeing. Puede resultar de especial importancia después de comprobada la efectividad del método, buscar patrones horarios y estacionales de la variación del seeing. 


\section{BIBLIOGRAFIA}

1. O'Byrne, J.W. Seeing Measurements Using a Shearing Interferometer. Publications of the Astronomical Society of the Pacific. 100:1169-1177. Septiembre 1988.

2. Tubbs, Robert Nigel. Lucky exposures: diffraction limited astronomical imaging through the atmosphere. St. Johns College, Cambridge University. Septiembre 2003. 